\title{
Hair dye-incorporated poly- $\gamma$-glutamic acid/glycol chitosan nanoparticles based on ion-complex formation
}

\section{Hye-Young Lee ${ }^{1, *}$ \\ Young-IL Jeong ${ }^{2, *}$ \\ $\mathrm{Ki}-$ Choon $\mathrm{Choi}^{3}$}

'Anyang Science University, Anyang, Gyeonggi, South Korea; ${ }^{2}$ Chonnam National University Hwasun Hospital, Jeonnam, South Korea; ${ }^{3}$ Grassland and Forages Research Center, National Institute of Animal Science, Rural Development Administration, Chungnam, South Korea

*These authors contributed equally to this work.
This article was published in the following Dove Press journal:

International Journal of Nanomedicine

16 November 2011

Number of times this article has been viewed

Background: $p$-Phenylenediamine (PDA) or its related chemicals are used more extensively than oxidative hair dyes. However, permanent hair dyes such as PDA are known to have potent contact allergy reactions in humans, and severe allergic reactions are problematic.

Methods: PDA-incorporated nanoparticles were prepared based on ion-complex formation between the cationic groups of PDA and the anionic groups of poly $(\gamma$-glutamic acid) (PGA). To reinforce PDA/PGA ion complexes, glycol chitosan (GC) was added. PDA-incorporated nanoparticles were characterized using field-emission scanning electron microscopy, Fourier-transform infrared (FT-IR) spectroscopy, dynamic light scattering, and powder X-ray diffractometry (XRD).

Results: Nanoparticles were formed by ion-complex formation between the amine groups of PDA and the carboxyl groups of PGA. PDA-incorporated nanoparticles are small in size $(<100 \mathrm{~nm})$, and morphological observations showed spherical shapes. FT-IR spectra results showed that the carboxylic acid peak of PGA decreased with increasing PDA content, indicating that the ion complexes were formed between the carboxyl groups of PGA and the amine groups of PDA. Furthermore, the intrinsic peak of the carboxyl groups of PGA was also decreased by the addition of GC. Intrinsic crystalline peaks of PDA were observed by XRD. This crystalline peak of PDA was completely nonexistent when nanoparticles were formed by ion complex between PDA, PGA, and GC, indicating that PDA was complexed with PGA and no free drug existed in the formulation. During the drug-release experiment, an initial burst release of PDA was observed, and then PDA was continuously released over 1 week. Cytotoxicity testing against $\mathrm{HaCaT}$ human skin keratinocyte cells showed PDA-incorporated nanoparticles had lower toxicity than PDA itself. Furthermore, PDA-incorporated nanoparticles showed reduced apoptosis and necrosis reaction at $\mathrm{HaCaT}$ cells.

Conclusion: The authors suggest that these microparticles are ideal candidates for a vehicle for decreasing side effects of hair dye.

Keywords: $p$-phenylenediamine, keratinocyte, PDA, PGA

\section{Introduction}

In recent decades, permanent hair dyes have received considerable attention due to their potent contact allergen $p$-phenylenediamine (PDA). PDA or its related chemicals are used more extensively than oxidative hair dyes. However, hypersensitivity, immune response, or severe allergic reactions following hair-dyeing have been reported. ${ }^{1}$ In particular, consumer-available PDA-containing hair dyes are known to be potent immune activators that lead to increased inflammatory cytokines. ${ }^{2}$ Furthermore, PDA may cause deoxyribonucleic acid (DNA) cleavage or gene toxicity against human skin keratinocyte cells under light irradiation. ${ }^{3}$ Derivatives of PDA chemicals are known to
Correspondence: Ki Choon Choi Grassland and Forages Research Center, National Institute of Animal Science, Rural Development Administration, Chungnam 33I-808, Korea

Tel $+824 I 5806752$

Email choiwh@rda.go.kr 
induce DNA damage and carcinogenic reactions in human skin keratinocyte cells., ${ }^{4,5}$

Nanoparticles have been extensively investigated to attain efficient drug delivery to a desired site of action and to control drug release for a desired length of time. ${ }^{6,7}$ Due to their small sizes, nanoparticles can be administered via various routes such as parenteral, oral, ocular, dermal or transdermal, and intranasal inhalation. ${ }^{8-14}$ Nanoparticles are regarded as ideal vehicles for targeted delivery to a specific body site and for reduction of unwanted side effects of bioactive agents. ${ }^{7-9}$ Particularly, nanoparticles are known to be efficient carriers for topical delivery of bioactive agents to the skin and hair. ${ }^{13,14}$ Lademann et al reported that dye-containing nanoparticles penetrate much deeper into the hair follicles and are stored in the hair follicles longer than the nonparticle form. ${ }^{14}$ Hair ingredient-encapsulated nanoparticles are known to enhance hair growth. ${ }^{15}$ Furthermore, the intrinsic small size of nanoparticles is regarded as playing an important role in skin penetration and dermal drug delivery. ${ }^{16}$

In this study, the formation of nanoparticles incorporating PDA was investigated. PDA-incorporated nanoparticles were prepared through ion-complex formation between hair dye and poly $(\gamma$-glutamic acid) (PGA)/chitosan. The physicochemical properties of PDA-incorporated nanoparticles were studied using dynamic light scattering (DLS), Fourier-transform infrared (FT-IR), powder X-ray diffractometry (XRD), and field-emission scanning electron microscopy (FE-SEM). Furthermore, the cytotoxicity of PDA-incorporated nanoparticles was tested using human skin keratinocyte cells.

\section{Materials and methods Materials}

PGA (molecular weight 200,000-500,000 g/mol) was purchased from WakoPure Chem Co (Osaka, Japan). Glycol chitosan (GC) was a product of MP Biomedicals, LLC (Santa Ana, CA). Propidium iodide (PI) and PDA were purchased from Sigma-Aldrich Co (St Louis, MO). Fluorescein isothiocyanate (FITC)-annexin V was obtained from Santa Cruz Biotechnology, Inc (Santa Cruz, CA).

\section{Preparation of PDA-incorporated nanoparticles}

PGA was distributed in $5 \mathrm{~mL}$ of deionized water and magnetically stirred for 30 minutes. PDA (5-40 mg) dissolved in $2 \mathrm{~mL}$ of deionized water was then added to this solution and stirred for 30 minutes. GC dissolved in $5 \mathrm{~mL}$ deionized water was added to this solution, and stirred for
30 minutes at room temperature. The resulting solutions were then lyophilized or analyzed.

\section{Characterization of PDA-incorporated nanoparticles}

Particle size of nanoparticles was measured with DLS (DLS7000; Otsuka Electronics Co, Osaka, Japan).

FT-IR spectroscopy (FT-IR 8700; Shimadzu, Osaka, Japan) was employed for ion-complex formation between PDA and polymers. PDA or lyophilized nanoparticles were used for analysis.

The morphology of the nanoparticles was observed with FE-SEM (S-4700; Hitachi Co Ltd, Tokyo, Japan). Microspheres were placed on double-sided tape attached to a graphite surface. Each sample was coated with gold/ palladium using an ion sputter (JFC-1100; Jeol Ltd, Tokyo, Japan). Coating was done at $20 \mathrm{~mA}$ for 4 minutes. Observation was done at $15 \mathrm{kV}$.

XRD diffractograms were employed to analyze crystallinity of drug and drug-incorporated nanoparticles using a Rigaku D/Max-1200 (Rigaku, Tokyo, Japan) equipped with Ni-filtered $\mathrm{Cu} \mathrm{K} \alpha$ radiation $(40 \mathrm{kV}, 20 \mathrm{~mA})$. The conditions of powder XRD measurement were as follows:

- Data type = binary; goniometer $=1$; attachment $=1$; scan mode $=$ continuous

- $\operatorname{Mode} 2(\mathrm{R} / \mathrm{T})=$ reflection; scan axis $=2 \theta / \theta$

- Start angle $=10,000$; stop angle $=80,000 ;$ scan speed $=5000 ;$ sampling interval $=0.050 ; \theta$ angle $=5000$; $2 \theta$ angle $=10,000$; fixed time $=0.01$; full scale $=1000$; counting unit $=\mathrm{CPS}$; target $=\mathrm{Cu}$

- Wavelength, $\mathrm{K} \alpha_{1}=1.540510$; wavelength, $\mathrm{K} \alpha_{2}=1.544330$; wavelength, $\mathrm{K} \alpha=1.541780$; wavelength, $\mathrm{K} \beta=1.392170$

- $40.0 \mathrm{kV} ; 20.0 \mathrm{~mA}$

PDA, PGA, GC, and PDA-incorporated nanoparticles were used to measure powder XRD. For physical mixture of empty nanoparticles and PDA, $90 \mathrm{mg}$ of empty nanoparticles was mixed with $10 \mathrm{mg}$ of PDA.

\section{Drug-release study}

The drug-release test was as follows: $5 \mathrm{mg}$ of PDAincorporated nanoparticles were redistributed in $5 \mathrm{~mL}$ of phosphate-buffered saline (PBS) (0.1 M, pH 7.4), and then this solution was placed in a dialysis tube. This dialysis tube was introduced into a $100 \mathrm{~mL}$ bottle with $95 \mathrm{~mL}$ of PBS (0.01 M, pH 7.4). At predetermined time intervals, whole media were taken and exchanged with fresh PBS. The concentration of released PDA was measured with a high performance liquid chromatography (HPLC) system 
as reported by Hooff et al. ${ }^{17}$ The FlexarTM HPLC system (PerkinElmer, Waltham, MA) consisted of a Solvent Manager 5-CH Degasser, an autosampler, a quaternary LC pump, a column oven, and an ultraviolet-visible detector. Gradient elution was performed as follows: elution solvent, $90 \%$ methanol with $10 \%$ water, and 0.01 $\mathrm{M}$ ammonium hydroxide ( $\mathrm{pH}$ 9). The gradient was initiated at $5 \%$ elution solvent for 4.5 minutes, and subsequently a linear gradient led to $95 \%$ elution solvent in 5 minutes, kept for 2 minutes, and brought back to $5 \%$ elution solvent in 0.5 minutes. A Luna phenyl-hexyl column from Phenomenex (Aschaffenburg, Germany) was kept at $20^{\circ} \mathrm{C}$ for the separation. PDA was monitored at wavelengths of $240 \mathrm{~nm}$ and $310 \mathrm{~nm}$.

\section{Cell culture}

For cytotoxicity testing of PDA and PDA-incorporated nanoparticles, HaCaT human keratinocyte cell line obtained from the Korean Cell Line Bank (Seoul, Korea) was maintained in Dulbecco's modified Eagle's medium (DMEM) medium at $37^{\circ} \mathrm{C}$ and $5 \% \mathrm{CO}_{2}$ atmosphere.

\section{MTT (3-[4,5-dimethylthiazol-2-yl]-2, 5-diphenyltetrazolium bromide) assay}

The medium for cell culture and proliferation inhibition assay was DMEM supplemented with $10 \%$ fetal bovine serum; cells were maintained in this medium in a $5 \% \mathrm{CO}_{2}$ incubator $\left(37^{\circ} \mathrm{C}\right)$. The cytotoxicity of PDA and PDAincorporated nanoparticles was determined using an MTT cell proliferation assay. HaCaT cells were seeded in 96-well plates at a density of $1 \times 10^{4}$ cells/well and incubated for 1 day in a $5 \% \mathrm{CO}_{2}$ incubator at $37^{\circ} \mathrm{C}$. PDA and PDAincorporated nanoparticles in deionized water were then diluted with serum-free DMEM media and then added to the $\mathrm{HaCaT}$ cells. The control was treated with serum-free DMEM media. Three days later, cell viability was assayed using the MTT colorimetric assay. After the incubation period, $30 \mu \mathrm{L}$ of MTT ( $5 \mathrm{mg} / \mathrm{mL}$ in PBS, pH 7.4) was added to each well and incubated for 4 hours. The formazan crystals were then solubilized with DMSO, and the absorbance (560 nm test/630 $\mathrm{nm}$ reference) was determined using an automated computer-linked microplate reader (Molecular Device Co, Sunnyvale, CA). Each experimental value was the mean of eight wells.

\section{Flow cytometry analysis}

Two reagents, PI and FITC-annexin V, were used to identify apoptosis and necrosis of HaCaT cells, respectively. Cells were treated with various concentrations of PDA and PDAincorporated nanoparticles for 24 hours. After that, cells were harvested and washed with PBS. The pellets were resuspended with binding buffer (HEPES [10 mM 4-(2hydroxyethyl)-1-piperazineethanesulfonic acid] $\mathrm{pH} 7.4$, $150 \mathrm{mM} \mathrm{NaCl}, 5 \mathrm{mM} \mathrm{KCl}, 1 \mathrm{mM} \mathrm{MgCl}, 1.8 \mathrm{mM} \mathrm{CaCl}_{2}$ ) containing FITC-annexin V $(1 \mu \mathrm{g} / \mathrm{mL})$ and further incubated for 30 minutes. Ten minutes prior to termination of incubation, PI $(10 \mu \mathrm{g} / \mathrm{mL})$ was added to stain necrotic cells under dark conditions. Following that, the cells were immediately analyzed using a FACScan (Becton Dickinson Biosciences, San Jose, CA) flow cytometer.

\section{Results and discussion Characterization of PDA-incorporated nanoparticles}

PDA-incorporated nanoparticles were prepared via ioncomplex formation between cationic groups of PDA and anionic groups of PGA (see Figure 1). As shown in Figure 1, when PGA was added to deionized water, it was not soluble in water. However, clear solution was obtained when PDA was added. This result might indicate that ion complexes were formed between the amine groups of PDA and the carboxyl groups of PGA.

Prior to studying its chemical properties, the potential of nanoparticle formation was investigated. To investigate its colloidal properties, DLS was used to test particle size and FE-SEM was used to observe morphology. As shown in Figure 2A, the particle size was about $76.9 \mathrm{~nm}$. Furthermore, spherical nanoparticles were observed during FE-SEM observation, as shown in Figure 2B. Chemical properties of PDA-incorporated nanoparticles were analyzed using FT-IR (see Figure 3). As shown in Figure 3, the primary amine peak and the carboxylic acid peak appeared for PDA and PGA, respectively. Interestingly, the intrinsic carboxylic acid peak of PGA was significantly decreased at peak analysis of nanoparticles. Furthermore, the primary amine peak of PDA was also decreased in its nanoparticles, indicating that ion complexes were formed between amine groups of PDA and carboxyl groups of PGA.

To reinforce PDA-incorporated nanoparticles, GC was added, as shown in Figure 4 (top pictures). Furthermore, changes of physicochemical properties were tested relative to the changes in PDA content and GC content, as shown in Figure 4 and Table 1. As shown in Figure 4, the opaque PGA solution became a brown, transparent solution after addition of PDA (Figure 4, top-left), indicating that the transparent solution was obtained by the formation of nanosized ion 


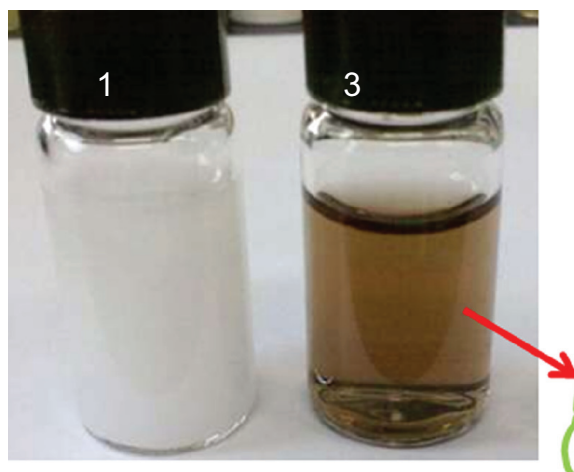

1. PGA in deionized water.

2. PDA was added to this solution.<smiles>Nc1ccc(N)cc1</smiles>

3. PDA/PGA polyelectrolyte complex.

Figure I Schematic illustrations of PDA-incorporated nanoparticles based on ion-complex formation between PDA and PGA. Weight ratio of PDA/PGA was 20/50 (mg/mg). The opaque solution of PGA was readily changed to a transparent solution by addition of PDA.

Abbreviations: PDA, p-phenylenediamine; PGA, poly $(\gamma$-glutamic acid $)$.

complexes. When GC was added, the particle size was increased (Table 1), and the nanoparticle solution became slightly turbid, as shown in Figure 4 (top-right).

As shown in Figure 5, the free carboxylic acid peak of PGA decreased with increase in PDA content, indicating that
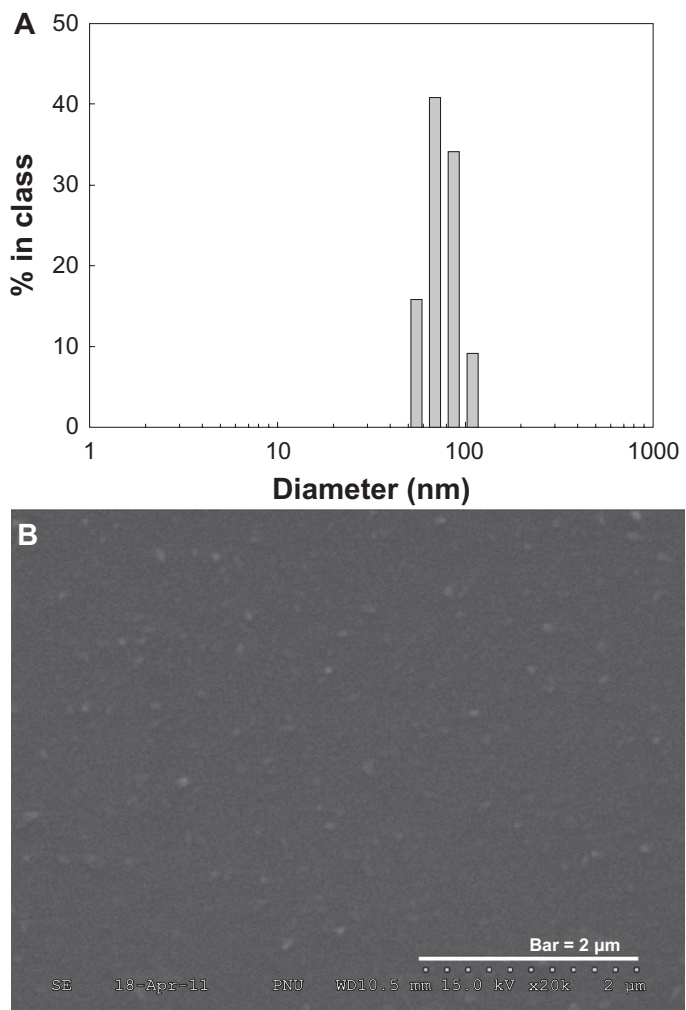

Figure 2 Typical particle size (A) and SEM photo (B) of PDA-incorporated PGA nanoparticles.

Note: Composition of PDA/PGA is PDA5 in Table 1.

Abbreviations: PDA, p-phenylenediamine; PGA, poly $(\gamma$-glutamic acid $)$; SEM, scanning electron microscopy. ion complexes between carboxyl groups and amine groups were increased and free carboxylic acid was decreased, as shown in Figure 6. At a fixed amount of PDA, particle size was not significantly changed by variations in the amount of GC, as shown in Table 1. The peak of free carboxylic acid of PGA also decreased with increasing GC content, indicating

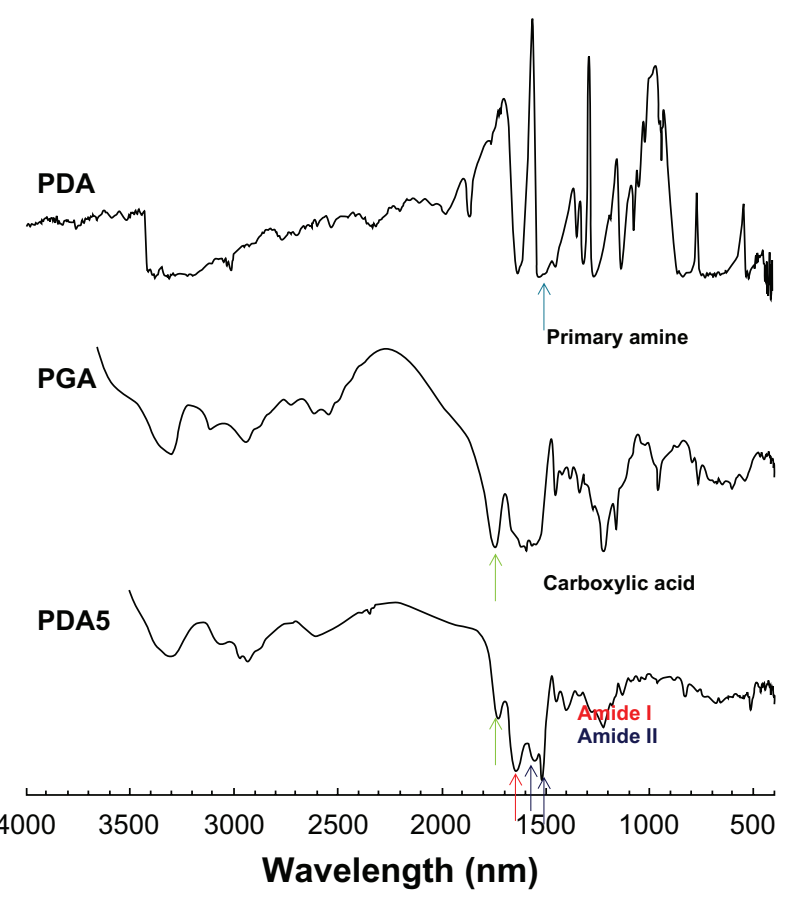

Figure 3 FT-IR analysis of PDA-incorporated nanoparticles.

Notes: PDA and PGA were used as a solid powder, and lyophilized PDA-incorporated nanoparticles (PDA5 in Table I) were used for FT-IR measurement. Carboxylic groups of PGA were decreased by complexation with PDA at PDA5 measurement. Abbreviations: FT-IR, Fourier-transform infrared; PDA, p-phenylenediamine; PGA, poly $(\gamma$-glutamic acid). 

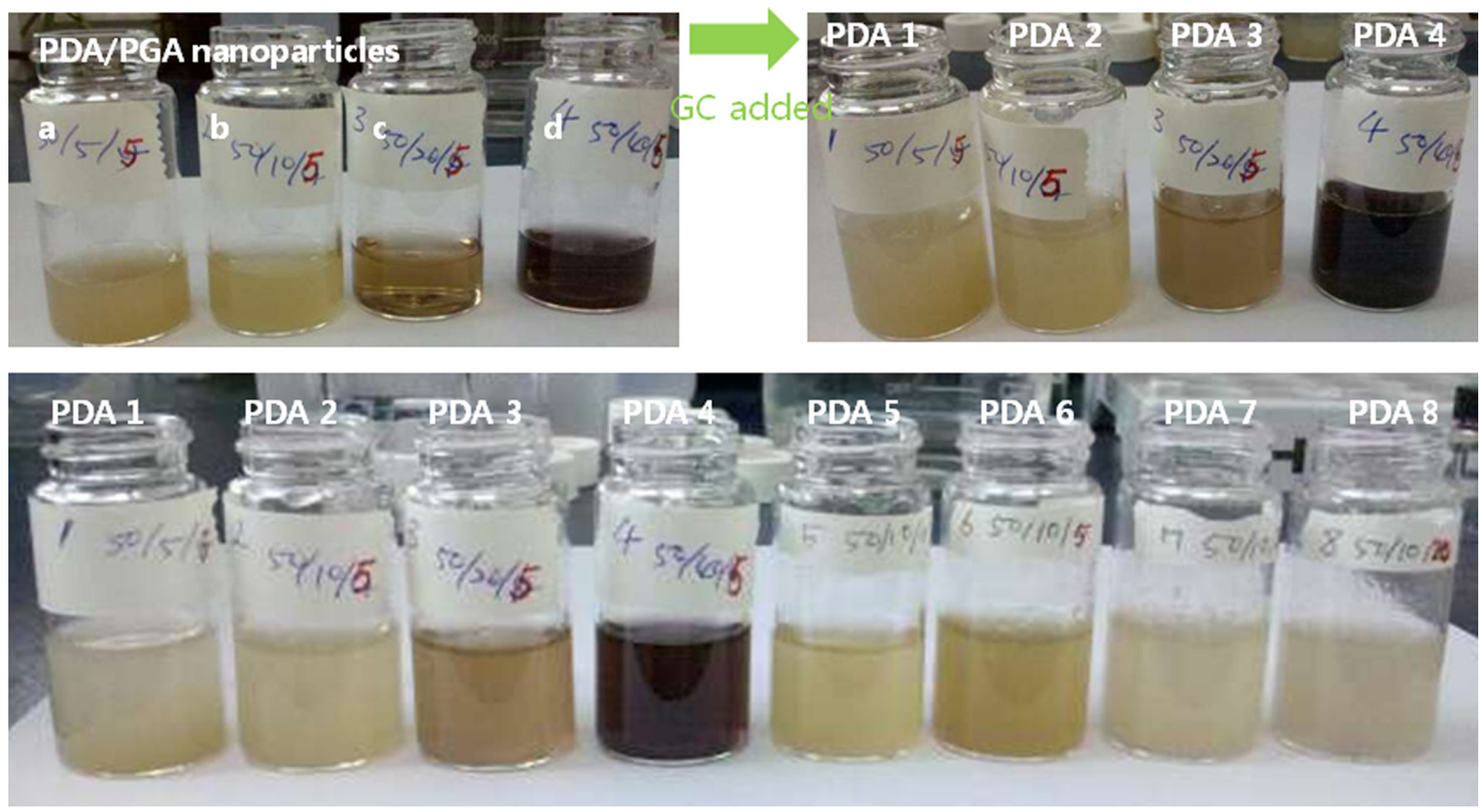

Figure 4 Photos of the PDA-incorporated nanoparticles. Photo in top-left box is of ion complexes of PDA-incorporated PGA nanoparticles: PDA/PGA weight ratio (w/w) (a) 50/5; (b) 50/10; (c) 50/20; and (d) 50/40. The top-right box is of nanoparticles after addition of GC, and the bottom photo is of PDA-incorporated nanoparticles. Note: The composition of PDA/PGA/GC is illustrated in Table I.

Abbreviations: GC, glycol chitosan; PDA, p-phenylenediamine; PGA, poly( $\gamma$-glutamic acid).

that ion complexes between amine groups of GC and carboxyl groups of PGA had increased.

To investigate the physical state of the nanoparticles, powder XRD was employed (see Figure 7). As shown in Figure 7, PDA had sharp crystalline peaks, while peaks of PGA and GC were relatively broad. Interestingly, crystalline peaks of PDA disappeared due to ion-complexes with PGA and nanoparticle formation, while physical mixtures of PDA and PGA still showed both sharp crystalline peaks of PDA and broad peaks of PGA. These results indicate that ion complexes between PDA, PGA, and GC were formed, and no large amount of free drug existed in the complexes with PGA. However, as shown in Figure 7, a small amount of free drug may have remained when PDA content was $40 \mathrm{mg}$ (PDA4 in Table 1 and Figure 7 [arrow]).

\section{Drug release from PDA-incorporated nanoparticles}

PDA release properties were investigated in vitro (see Figure 8). As shown in Figure 8A, PDA was continuously released over 1 week. PDA release rate increased with escalating PDA content. These results may be due to PDA being liberated at higher drug contents and being decomplexed easily from the nanocomplexes due to competition

Table I Characterization of PDA-incorporated submicroparticles of PGA/GC complexes

\begin{tabular}{|c|c|c|c|c|c|c|}
\hline \multirow[t]{2}{*}{ Formulation no. } & \multirow{2}{*}{$\begin{array}{l}\text { Weight ratio of } \\
\text { PGA/PDA/GC }(\mathrm{mg})^{a}\end{array}$} & \multirow{2}{*}{$\begin{array}{l}\text { Drug contents } \\
(\%, w / w)\end{array}$} & \multicolumn{3}{|l|}{ Particle size (nm) } & \multirow[t]{2}{*}{ Status $^{b}$} \\
\hline & & & Intensity average & Weight average & Number average & \\
\hline PDAI & $50: 5: 5$ & 8.3 & $253.8 \pm 108.5$ & $242.3 \pm 78.5$ & $230.2 \pm 61.8$ & + \\
\hline PDA2 & $50: 10: 5$ & 15.4 & $336.2 .4 \pm 105.5$ & $313.4 \pm 90.3$ & $280.9 \pm 81.2$ & + \\
\hline PDA3 & $50: 20: 5$ & 26.7 & $554.3 \pm 130.5$ & $536.4 \pm 121.2$ & $465.7 \pm 110.8$ & ++ \\
\hline PDA4 & $50: 40: 5$ & 42.1 & $689.6 \pm 121.4$ & $680.0 \pm 115.8$ & $523.5 \pm 98.7$ & ++ \\
\hline PDA5 & $50: 10: 0$ & 16.7 & $121.3 \pm 60.9$ & $117.9 \pm 58.7$ & $76.9 \pm 42.0$ & - \\
\hline PDA6 & $50: 10: 5$ & 15.4 & $312.5 \pm 103.7$ & $290.6 \pm 93.6$ & $262.4 \pm 63.7$ & + \\
\hline PDA7 & $50: 10: 10$ & 14.3 & $320.7 \pm 96.7$ & $298.9 \pm 102.8$ & $278.6 \pm 82.6$ & ++ \\
\hline PDA8 & $50: 10: 20$ & 12.5 & $328.7 \pm 150.9$ & $295.6 \pm 121.3$ & $281.8 \pm 102.9$ & +++ \\
\hline
\end{tabular}

Notes: a Final volume of nanoparticle solution was adjusted to $10 \mathrm{~mL}$; ${ }^{b}$ fine distribution $(+$, small amount of aggregates; ++ , moderate amount of aggregates; +++ , significant amount of aggregates or precipitation).

Abbreviations: GC, glycol chitosan; PDA, p-phenylenediamine; PGA, poly $(\gamma$-glutamic acid). 

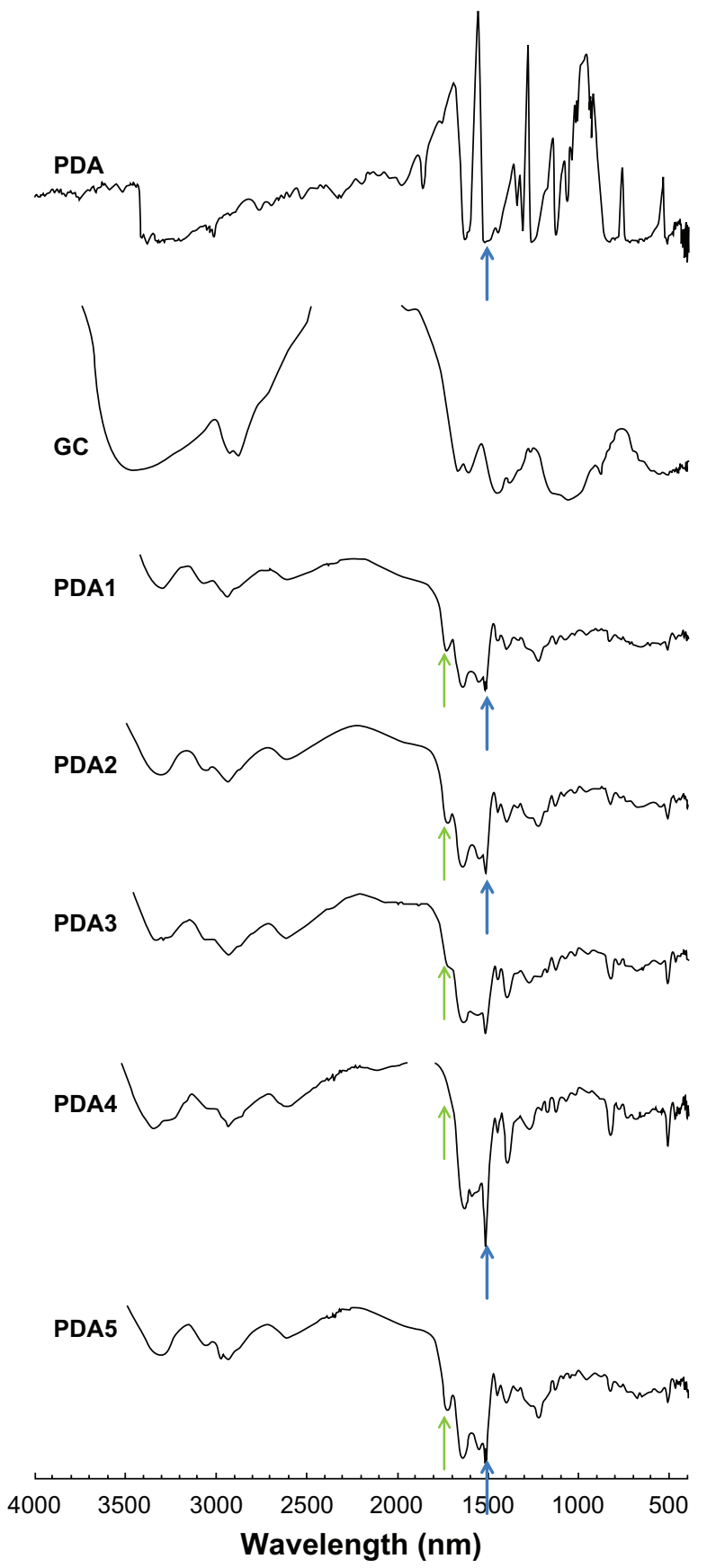

Figure 5 FT-IR analysis of PDA-incorporated nanoparticles, showing the effect of PDA content on the chemical properties of nanoparticles.

Notes: The composition of PDA/PGA/GC is illustrated in Table I. PDA, PGA, and GC were used as a solid powder, and lyophilized PDA-incorporated nanoparticles (PDAI-5 in Table I) were used for FT-IR measurement. As PDA content increased, the carboxylic acid peak of PGA gradually decreased.

Abbreviations: GC, glycol chitosan; FT-IR, Fourier-transform infrared; PDA, p-phenylenediamine; PGA, poly $(\gamma$-glutamic acid).

with each other. As shown in Figure 8B, the PDA release rate was also increased by the addition of GC. These results indicated that the increase in the amount of amine groups may enhance competition in the ion complexes with carboxylic acid, and this fact may increase release rate. For all samples, the initial burst release was observed until
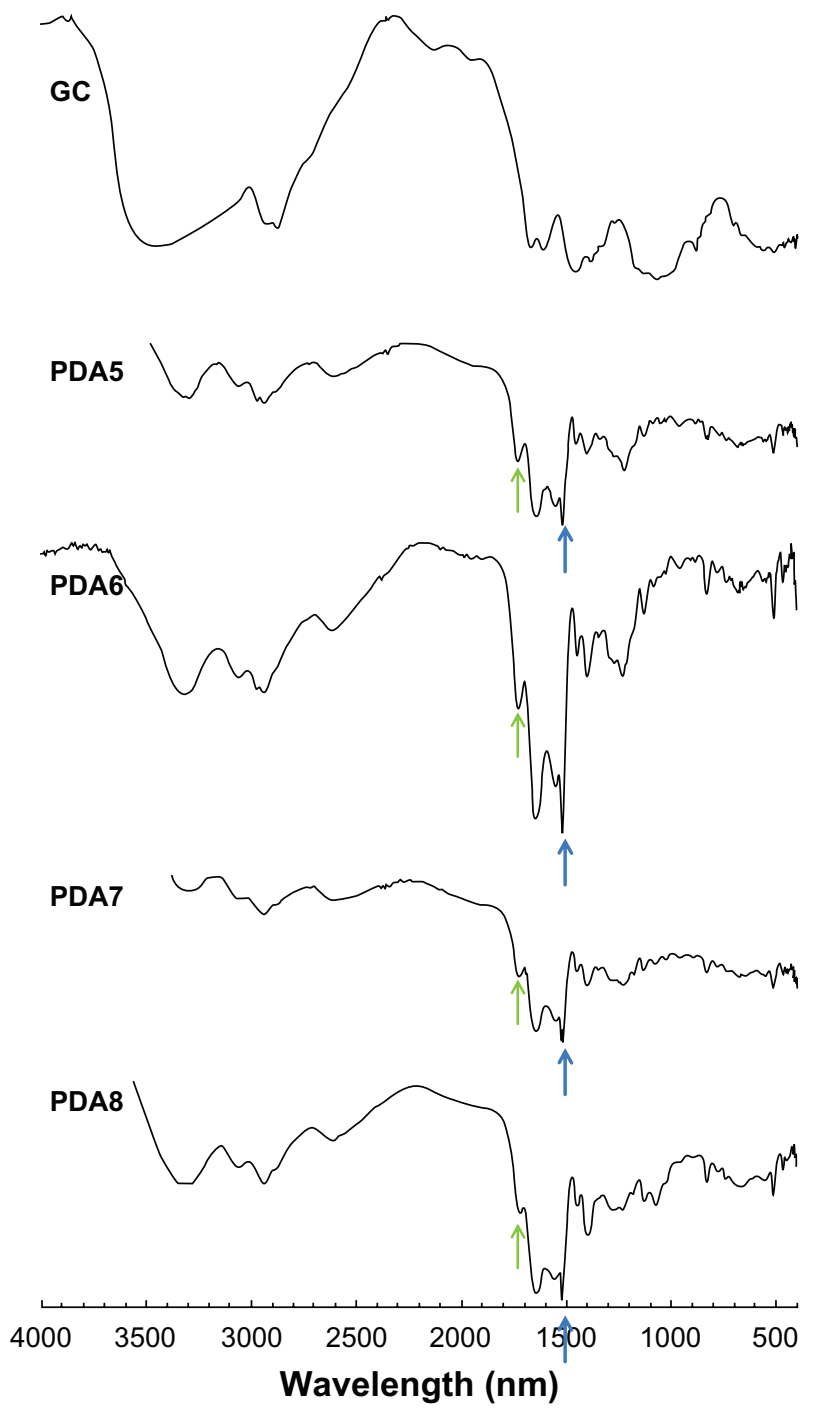

Figure 6 FT-IR analysis of PDA-incorporated nanoparticles showing the effect of GC addition on the chemical properties of nanoparticles.

Notes: The composition of PDA/PGA/GC is illustrated in Table I. GC was used as a solid powder, and lyophilized PDA-incorporated nanoparticles (PDA5-8 in Table I) were used for FT-IR measurement. The carboxylic acid peak of PGA decreased with increasing GC content.

Abbreviations: GC, glycol chitosan; FT-IR, Fourier-transform infrared; PDA, p-phenylenediamine; PGA, poly $(\gamma$-glutamic acid).

6 hours, after which time, the PDA release rate was relatively sustained.

At the next step, formulation PDA2 (same as PDA6) was used because the aqueous solution of PDA2 was relatively stable compared with the other formulations, and its drugrelease kinetics were relatively sustained.

\section{Cell cytotoxicity of PDA-incorporated nanoparticles}

To investigate cytotoxicity of PDA and PDA-incorporated nanoparticles, $\mathrm{HaCaT}$ human skin keratinocyte cells were employed, and cytotoxicity was evaluated using MTT cell proliferation assay. As shown in Figure 9, cell viability 

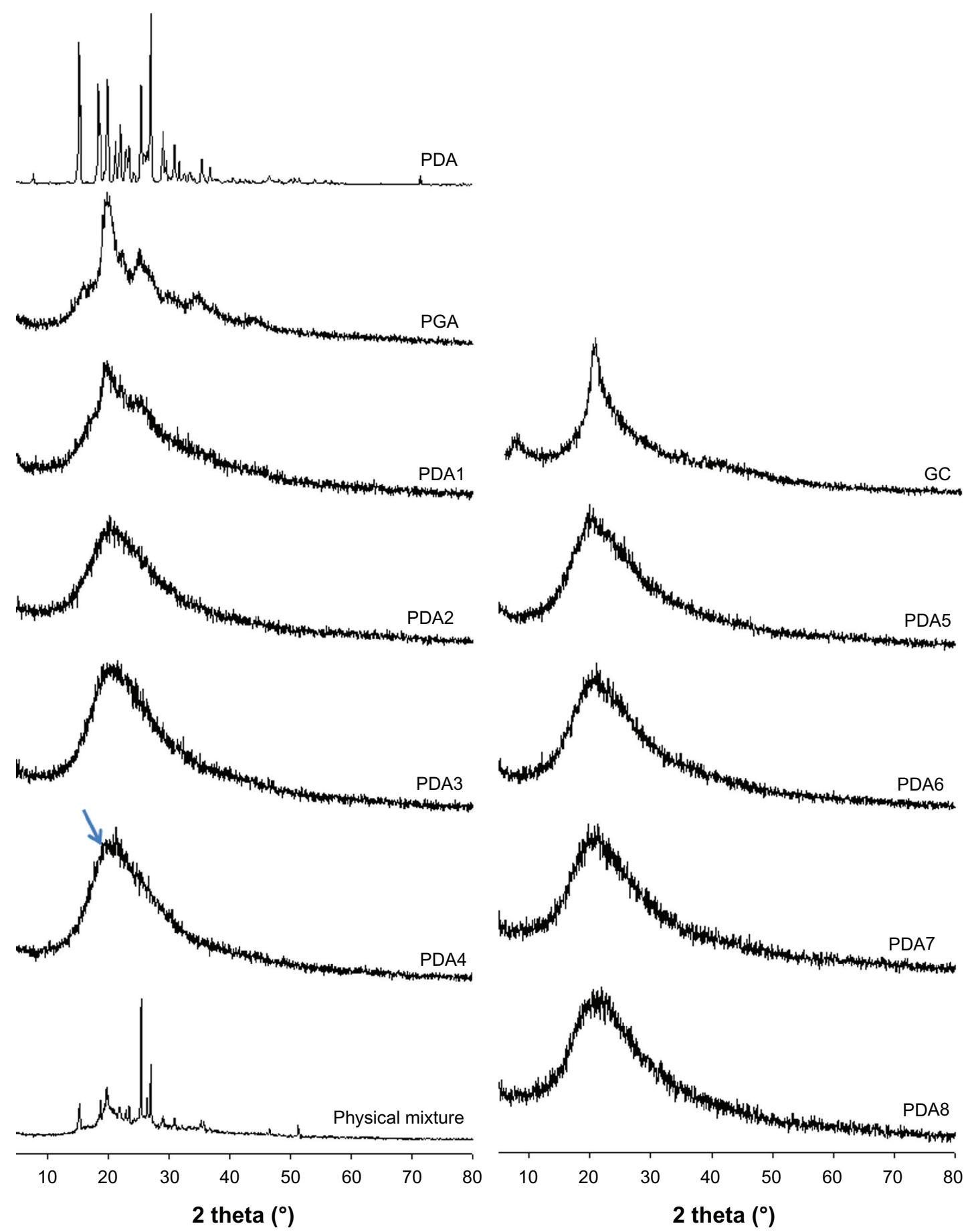

Figure 7 XRD analysis of PDA-incorporated nanoparticles.

Notes: The composition of PDA/PGA/GC is illustrated in Table I. PDA, PGA, and GC were used as a solid powder, and lyophilized PDA-incorporated nanoparticles (PDAI-8 in Table I) were used for XRD analysis. The physical mixture was PGA/PDA (weight ratio = 5:I). PDA itself has sharp crystalline peaks, while nanoparticles showed broad peaks for all compositions.

Abbreviations: GC, glycol chitosan; PDA, p-phenylenediamine; PGA, poly ( $\gamma$-glutamic acid); XRD, X-ray diffractometry.

was decreased in a dose-dependent manner when $\mathrm{HaCaT}$ cells were exposed to PDA itself. On the other hand, when $\mathrm{HaCaT}$ cells were exposed to PDA-incorporated nanoparticles, viability of cells was not significantly decreased. These results indicated that intrinsic cytotoxicity of PDA was alleviated. Although the reason for these results was not clear, the sustained-release properties of the nanoparticles might be affected by the alleviated toxicity, and another possibility is better biocompatibility of PGA/GC nanoparticles compared with PDA itself. The mechanistic study of cellular cytotoxicity was investigated using Annexin V staining for apoptosis analysis and PI staining for necrosis analysis. 

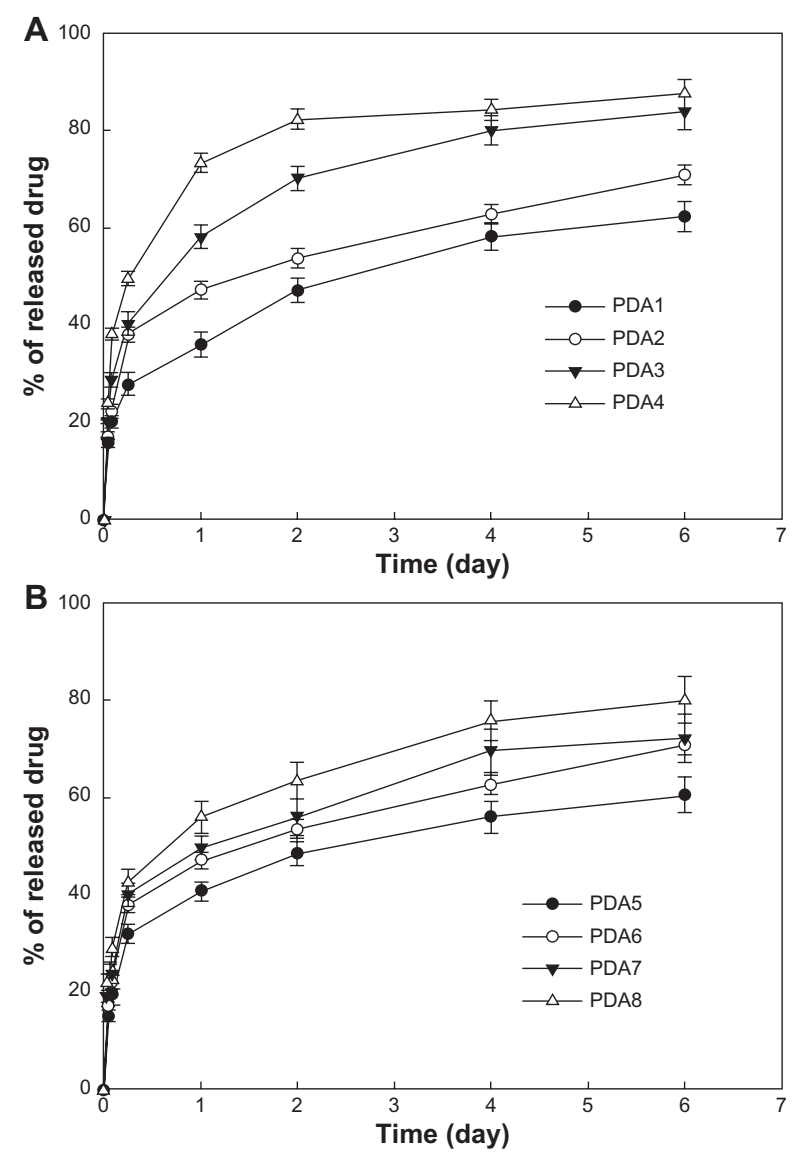

Figure 8 PDA release from nanoparticles: the effects of (A) PDA content and (B) the addition of GC.

Note: The composition of PDA/PGA/GC is illustrated in Table I.

Abbreviations: GC, glycol chitosan; PDA, $p$-phenylenediamine; PGA, poly $(\gamma$-glutamic acid).

As shown in Figure 10, both apoptosis and necrosis at PDA treatment was higher than nanoparticle treatment, indicating that nanosized ion-complex formation between PDA and ionic polymer decreased intrinsic cytotoxicity of PDA.

\section{Discussion}

Nanoparticles have been extensively investigated in the biomedical, cosmetic, and health industries. ${ }^{7-21}$ In particular, cosmetic application of nanoparticles is regarded as the best technology to upgrade the performance of cosmetic products and to meet the consumer's needs. ${ }^{19}$ For example, Popov et al showed that potential application of zinc oxide or titanium oxide nanoparticles for sunscreen has benefit to attenuate ultraviolet light by absorption and scattering. ${ }^{20}$ Furthermore, the effect of particle size on the potential of titanium oxide nanoparticles as a sunscreen was also reported by other researchers. ${ }^{22}$ Even though intrinsic toxicity was problematic, ${ }^{23}$ ingredients based on nanotechnology in cosmetic products are known to have no more risk to human health than larger-sized counterparts. ${ }^{21}$

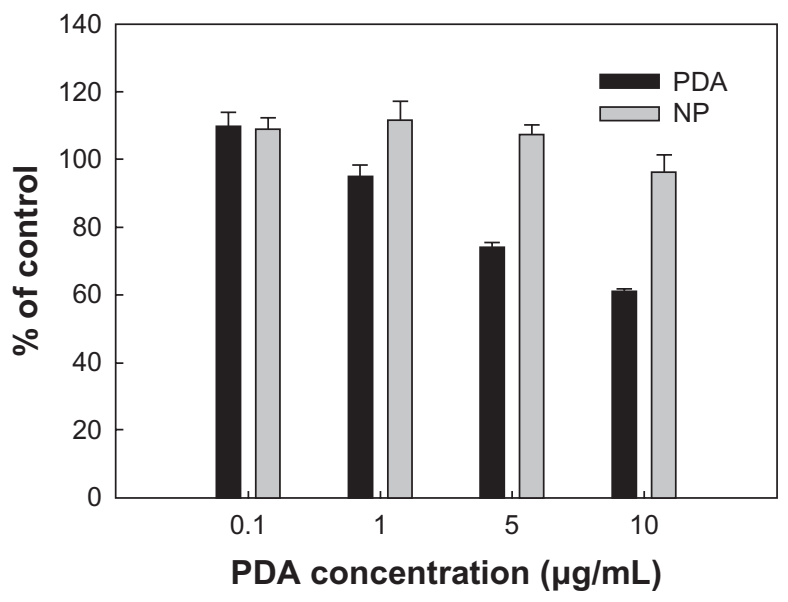

Figure 9 Cytotoxicity of PDA and PDA-incorporated nanoparticles (PDA2 in Table I) against $\mathrm{HaCaT}$ cells. $\mathrm{HaCaT}$ cells $\left(\mathrm{I} \times 10^{4}\right.$ cells $)$ were seeded in 96-well plates, and then the cells were exposed to PDA or PDA-incorporated nanoparticles for 3 days. The cell viability was assayed using the MTT colorimetric assay. Abbreviations: MTT, 3-(4,5-dimethylthiazol-2-yl)-2,5-diphenyltetrazolium bromide; NP, PDA-incorporated nanoparticles; PDA, p-phenylenediamine.

Due to hypersensitivity, allergy reaction, and immune response, permanent hair dyes such as PDA and its related chemicals have been widely investigated. ${ }^{1-5}$ Since PDA or its related chemicals are used more extensively than oxidative hair dyes, their immune-toxicity, carcinogenesis, and genetic toxicity is problematic to human skin keratinocyte cells. ${ }^{4,5}$ Mosley-Foreman et $\mathrm{al}^{3}$ reported that permanent hair dye revealed cytotoxicity against human skin keratinocyte cells under light irradiation, and no chemical structure-related phototoxicity of derivatives of PDA was found.

This current report describes the preparation of PDAincorporated nanoparticles using PGA and GC. PDA itself did not form nanoparticles in aqueous solutions (data not shown). Furthermore, PGA itself did not form nanoparticles, and PGA was not clearly dissolved in deionized water, ie, it was simply distributed in the deionized water. However, opaque PGA solution was readily changed into a transparent solution when PDA was added, as shown in Figure 1. Interestingly, PGA/PDA mixtures were found to form nanoparticles in the aqueous solution based on ion-complex formation between anionic groups of PGA and cationic groups of PDA. Since the formation of these nanoparticles did not require organic solvent or surfactant, nanoparticles based on ion complex between PGA and PDA are very interesting. Nanocomplexes based on polyelectrolytes between anionic compounds and cationic compounds have been extensively investigated by several researchers. ${ }^{24-28}$ Thünemann and Beyermann reported that retinoic acid as an anionic compound can form ion complexes with cationic polymers such as polyethyleneimine, and these complexes are nanosized carriers. ${ }^{24}$ Furthermore, they also reported that another cationic polymer, poly(ethylene 

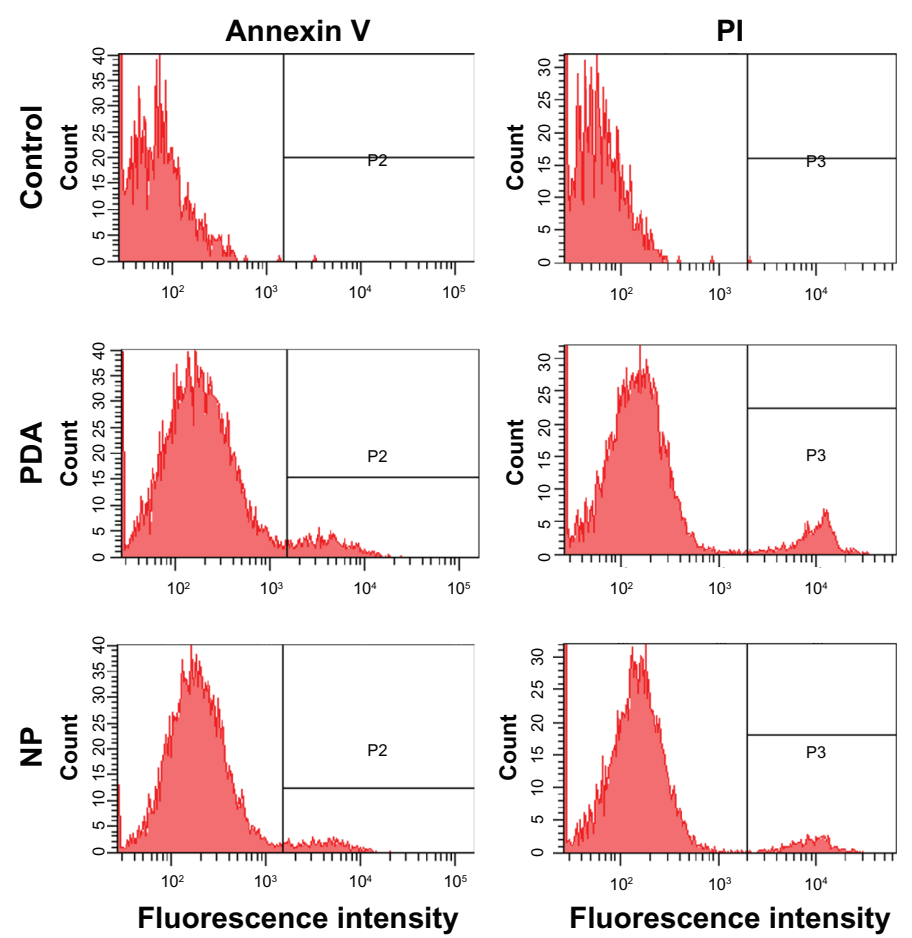

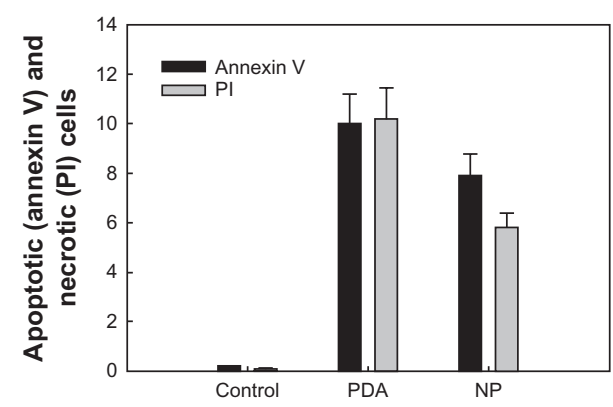

Figure 10 Flow cytometry analysis of PDA and PDA-incorporated nanoparticles (PDA2 in Table I) against HaCaT cells. FITC-annexin V (Annexin V) and PI were used for apoptosis and necrosis of cells, respectively. Apoptosis or necrosis of cells were analyzed using a FACScan (Becton Dickinson Biosciences, San Jose, CA) flow cytometer. Abbreviations: FITC, fluorescein isothiocyanate; NP, PDA-incorporated nanoparticles; PDA, p-phenylenediamine; PI, propidium iodide.

glycol)/poly(L-lysine) block copolymer, was also employed to form polyion complex micelles. Jeong et al reported that retinoic acid and chitosan can form polyion complex micelles via interaction between amine groups of chitosan and carboxyl groups of retinoic acid, while chitosan itself does not form polymeric micelles. ${ }^{26}$ In another report, they described nanoparticle formation based on ion complexes between hyaluronic acid and cisplatin. ${ }^{27}$ Furthermore, they also reported that carboxymethyl (CM) chitosan can form nanoparticles via ion-complex formation between carboxyl groups of CM chitosan and amine groups of doxorubicin. ${ }^{28}$ As shown in Figures 2-7, the results of this present study showed that PDA-incorporated nanoparticles can be formed by ion complexes between amine groups of PDA and carboxyl groups of PGA. The complexes formed nanoparticles of small size $(<1000 \mathrm{~nm})$ and spherical shape. Furthermore, intrinsic cytotoxicity of PDA can be reduced by nanoparticle formation, as shown in Figures 9 and 10. Even though initial burst release of PDA was observed, as shown in Figure 8, PDA-incorporated nanoparticles showed sustained release of PDA over 1 week. Even though the reason for the initial burst release of PDA up to 6 hours is not clear at this moment, we assumed that some of the PDA was weakly attached on the surface of the nanoparticles and then these drugs were liberated rapidly. Another reason for this initial-burst phenomenon might be due to the existence of free drug on the surface of the nanoparticles at higher drug concentrations, as shown in Figure 7 (arrow, PDA4). However, no drug crystalline peaks were observed for the other formulations. Therefore, the initial burst release from the nanoparticles must be due to the weak interaction between the drug and the polymer at the nanoparticle surface rather than the existence of free drug. White et al reported that intermittent exposure to low-concentration PDA can be equivalent to a single, higher-dose exposure. ${ }^{29}$ The authors of this present paper suggest that PDA-incorporated nanoparticles may reduce intrinsic toxicity of PDA and enhance potential application in the cosmetic industry.

\section{Conclusion}

PDA-incorporated nanoparticles were prepared based on ion-complex formation between cationic groups of PDA and anionic groups of PGA. To reinforce PDA/PGA ion complexes, GC was added. PDA-incorporated nanoparticles are small in size ( $<100 \mathrm{~nm}$ ), and morphological observations showed spherical shapes. FT-IR spectra showed the carboxylic acid peak of PGA decreased with increasing PDA content, indicating that ion complexes were formed between carboxyl groups of PGA and amine groups of PDA. XRD showed intrinsic crystalline peaks of PDA completely disappeared when nanoparticles were formed by ion complexes between PDA, PGA, and GC, indicating that PDA was complexed 
with PGA and no free drug existed in the formulation. The drug-release experiment showed an initial burst release of PDA and then continuous release over 1 week. Cytotoxicity of PDA-incorporated nanoparticles against HaCaT human skin keratinocyte cells was lower than PDA alone. Furthermore, PDA-incorporated nanoparticles showed reduced apoptosis and necrosis reaction in $\mathrm{HaCaT}$ cells.

\section{Acknowledgment}

This work was partially supported by a Grant-in-Aid (No. PJ007538) for scientific research from the Ministry for Food, Agriculture, Forestry and Fisheries of Korea.

\section{Disclosure}

The authors report no conflicts of interest in this work.

\section{References}

1. Goebel C, Coenraads PJ, Rothe H, et al. Elicitation of the immune response to $p$-phenylenediamine in allergic patients: the role of dose and exposure time. Br J Dermatol. 2010;163:1205-1211.

2. Rubin IM, Dabelsteen S, Nielsen MM, et al. Repeated exposure to hair dye induces regulatory T cells in mice. Br J Dermatol. 2010;163: 992-998.

3. Mosley-Foreman C, Choi J, Wang S, Yu H. Phototoxicity of phenylenediamine hair dye chemicals in Salmonella typhimurium TA102 and human skin keratinocytes. Food Chem Toxicol. 2008;46:3780-3784.

4. Murata M, Nishimura T, Chen F, Kawanishi S. Oxidative DNA damage induced by hair dye components ortho-phenylenediamines and the enhancement by superoxide dismutase. Mutat Res. 2006;607: 184-191.

5. Chen F, Murata M, Hiraku Y, Yamashita N, Oikawa S, Kawanishi S. DNA damage induced by m-phenylenediamine and its derivative in the presence of copper ion. Free Radic Res. 1998;29:197-205.

6. Kreuter J. Possibilities of using nanoparticles as carriers for drugs and vaccines. J Microencapsul. 1988;5:115-127.

7. Gref R, Minamitake Y, Peracchia MT, Trubetskoy V, Torchilin V, Langer R. Biodegradable long-circulating polymeric nanospheres. Science. 1994;263:1600-1603.

8. Gulati N, Gupta H. Parenteral drug delivery: a review. Recent Pat Drug Deliv Formul. 2011;5:133-145.

9. Takeuchi H, Sugihara H. Absorption of calcitonin in oral and pulmonary administration with polymer-coated liposomes. Yakugaku Zasshi. 2010; 130:1135-1142.

10. Schroeter A, Engelbrecht T, Neubert RH, Goebel AS. New nanosized technologies for dermal and transdermal drug delivery. A review. J Biomed Nanotechnol. 2010;6:511-528.

11. du Toit LC, Pillay V, Choonara YE, Govender T, Carmichael T. Ocular drug delivery - a look towards nanobioadhesives. Expert Opin Drug Deliv. 2011;8:71-94.
12. Alvarez-Román R, Naik A, Kalia YN, Guy RH, Fessi H. Enhancement of topical delivery from biodegradable nanoparticles. Pharm Res. 2004; 21:1818-1825.

13. Alvarez-Román R, Naik A, Kalia YN, Guy RH, Fessi H. Skin penetration and distribution of polymeric nanoparticles. J Control Release. 2004;99:53-62.

14. Lademann J, Richter H, Teichmann A, et al. Nanoparticles - an efficient carrier for drug delivery into the hair follicles. Eur J Pharm Biopharm. 2007;66:159-164.

15. Tsujimoto H, Hara K, Tsukada Y, et al. Evaluation of the permeability of hair growing ingredient encapsulated PLGA nanospheres to hair follicles and their hair growing effects. Bioorg Med Chem Lett. 2007;17: 4771-4777.

16. Schneider M, Stracke F, Hansen S, Schaefer UF. Nanoparticles and their interactions with the dermal barrier. Dermatoendocrinol. 2009;1: 197-206.

17. Hooff GP, van Huizen NA, Meesters RJ, et al. Analytical investigations of toxic $p$-phenylenediamine (PPD) levels in clinical urine samples with special focus on MALDI-MS/MS. PLoS One. 2011;6:e22191.

18. Singh R, Nalwa HS. Medical applications of nanoparticles in biological imaging, cell labeling, antimicrobial agents, and anticancer nanodrugs. J Biomed Nanotechnol. 2011;7:489-503.

19. Wiechers JW, Musee N. Engineered inorganic nanoparticles and cosmetics: facts, issues, knowledge gaps and challenges. $J$ Biomed Nanotechnol. 2010;6:408-431.

20. Popov AP, Zvyagin AV, Lademann J, et al. Designing inorganic light-protective skin nanotechnology products. J Biomed Nanotechnol. 2010;6:432-451.

21. Epstein HA. Nanotechnology in cosmetic products. Skinmed. 2011;9: 109-110.

22. Wokovich A, Tyner K, Doub W, Sadrieh N, Buhse LF. Particle size determination of sunscreens formulated with various forms of titanium dioxide. Drug Dev Ind Pharm. 2009;35:1180-1189.

23. Kong B, Seog JH, Graham LM, Lee SB. Experimental considerations on the cytotoxicity of nanoparticles. Nanomedicine (Lond). 2011;6: 929-941.

24. Thünemann AF, Beyermann J. Polyethyleneimine complexes with retinoic acid: structure, release profiles, and nanoparticles. Macromolecules. 2000;33:6878-6885.

25. Thünemann AF, Beyermann J, Kukula H. Poly(ethylene oxide)b-poly(L-lysine) complexes with retinoic acid. Macromolecules. 2000; 33:5906-5911.

26. Jeong YI, Kim SH, Jung TY, et al. Polyion complex micelles composed of all-trans retinoic acid and poly(ethylene glycol)-grafted-chitosan. J Pharm Sci. 2006;95:2348-2360.

27. Jeong YI, Kim ST, Jin SG, et al. Cisplatin-incorporated hyaluronic acid nanoparticles based on ion-complex formation. J Pharm Sci. 2008;97:1268-1276.

28. Jeong YI, Jin SG, Kim IY, et al. Doxorubicin-incorporated nanoparticles composed of poly(ethylene glycol)-grafted carboxymethyl chitosan and antitumor activity against glioma cells in vitro. Colloids Surf B Biointerfaces. 2010;79:149-155.

29. White JM, Basketter DA, Pease CK, Sanders DA, McFadden JP. Intermittent exposure to low-concentration paraphenylenediamine can be equivalent to single, higher-dose exposure. Contact Dermatitis. 2007; $56: 262-265$.

\section{Publish your work in this journal}

The International Journal of Nanomedicine is an international, peerreviewed journal focusing on the application of nanotechnology in diagnostics, therapeutics, and drug delivery systems throughout the biomedical field. This journal is indexed on PubMed Central, MedLine, CAS, SciSearch $\AA$, Current Contents ${ }^{\circledR} /$ Clinical Medicine,

\section{Dovepress}

Journal Citation Reports/Science Edition, EMBase, Scopus and the Elsevier Bibliographic databases. The manuscript management system is completely online and includes a very quick and fair peer-review system, which is all easy to use. Visit http://www.dovepress.com/ testimonials.php to read real quotes from published authors. 